\title{
Pulmonary aspiration occurring during the induction of anesthesia in a patient with esophageal dilatation
}

\author{
Hyun Kyoung Lim ${ }^{1}$, Mi Hyun Lee ${ }^{2}$, Chan Ik Jin ${ }^{1}$, Hyo Jin Byeon ${ }^{1}$, and Jang Ho Song ${ }^{1}$ \\ Department of Anesthesiology and Pain Medicine, ${ }^{1}$ Inha University College of Medicine, Incheon, ${ }^{2}$ Kangnam Sacred Heart Hospital, \\ Hallym University College of Medicine, Seoul, Korea
}

In recent years, pulmonary aspiration of gastro-esophageal contents during the induction of general anesthesia (GA) in Nil per Os (NPO) patients has become a rare complication. A retrospective study from the Mayo Clinic between 1985 and 1991 reported that the incidence of peri-operative pulmonary aspiration under GA for elective surgery was 1 in 3,886 cases [1]. Due to the low incidence rate of peri-operative pulmonary aspiration, precautions to prevent pulmonary aspiration are progressively being overlooked. However, pulmonary aspiration is closely related to postoperative mortality and pulmonary morbidity. Anesthesiologist should pay particular attention to patients who are at risk for pulmonary aspiration.

We present a patient with esophageal dilatation who suffered from pulmonary aspiration during induction of anesthesia. A 42-year old female who was suspected of having sarcoidosis was scheduled for mediastinal lymph node biopsy under GA for a conclusive diagnosis. Preoperative computed tomography (CT) of the chest showed dilatation of the esophagus with an air-fluid level indicating remnant food material leading to a suspicion of achalasia. The preoperative interview, with the exception of feeling a little heavy after meals, showed no history of dysphagia, regurgitation, nausea, prior vomiting or chest pain. No other abnormal findings were revealed through a physical examination. No premedication was given. With a fasting period of over 11 hours, denitrogenation with oxygen was performed for 3 minutes followed by intravenous injection with propofol $2 \mathrm{mg} / \mathrm{kg}$, vecuronium $0.1 \mathrm{mg} / \mathrm{kg}$ and lidocaine $60 \mathrm{mg}$. During assisted ventilation using sevoflurane 3.0 vol\% with $100 \%$ oxygen, abrupt resistance to ventilation was felt and the capnogram was flattened. When $\mathrm{O}_{2}$ saturation reached $65 \%$, endotracheal intubation was hastened and successfully executed. Abundant thick jelly-like salivary material was removed from the oral cavity through oral suction. After intubation, both lung sounds were barely heard through auscultation. After visual confirmation of the placement of the endotracheal tube, endotracheal suction was attempted removing material looking like pieces of bread, after which, $\mathrm{O}_{2}$ saturation improved to $97 \%$ and the patient's blood pressure was 147/86 mmHg with a heart rate of 99 beats/min. A 16-Fr naso-gastric tube was inserted and a small amount of foreign material was aspirated and the $\mathrm{pH}$ was 5.0. After resuming mechanical ventilation, the blood gas analysis showed pH 7.41, $\mathrm{PCO}_{2} 35 \mathrm{mmHg}$ and $\mathrm{PO}_{2} 257 \mathrm{mmHg}$ at a fraction of oxygen $\left(\mathrm{FiO}_{2}\right)$ at 1.0. Portable chest X-ray showed an increased shadow in the right upper lobe. The patient was awaken and then asked to cough with repeated endotracheal suction. Immediate consultation to the respiratory disease division was made and after removal of most of the foreign substances using a fiber-optic bronchoscope (FOB), anesthesia was resumed for the mediastinal biopsy. At a $\mathrm{FiO}_{2}$ of $0.5, \mathrm{O}_{2}$ saturation was maintained at $100 \%$, body temperature was $38.4^{\circ} \mathrm{C}$ and the other vital signs were stable. Postoperative chest $\mathrm{X}$-ray showed the improved status of the right upper lobe.

Achalasia is a motor disorder caused by the loss of intramural neuron of the esophageal smooth muscle. It is characterized by incomplete relaxation of the lower esophageal sphincter and a lack of peristaltic contractions in response to swallowing. The main symptom of achalasia is progressive difficulty in swallowing. Ingested food and saliva are retained in the

Corresponding author: Hyun Kyoung Lim, M.D., Department of Anesthesiology and Pain Medicine, Inha University Hospital, 7-206, \#3 Sinheung-dong, Jung-gu, Incheon 400-711, Korea. Tel: 82-32-890-3968, Fax: 82-32-881-2476, E-mail: hkliman@inha.ac.kr

(c) This is an open-access article distributed under the terms of the Creative Commons Attribution Non-Commercial License (http:// creativecommons.org/licenses/by-nc/3.0/), which permits unrestricted non-commercial use, distribution, and reproduction in any medium, provided the original work is properly cited. 


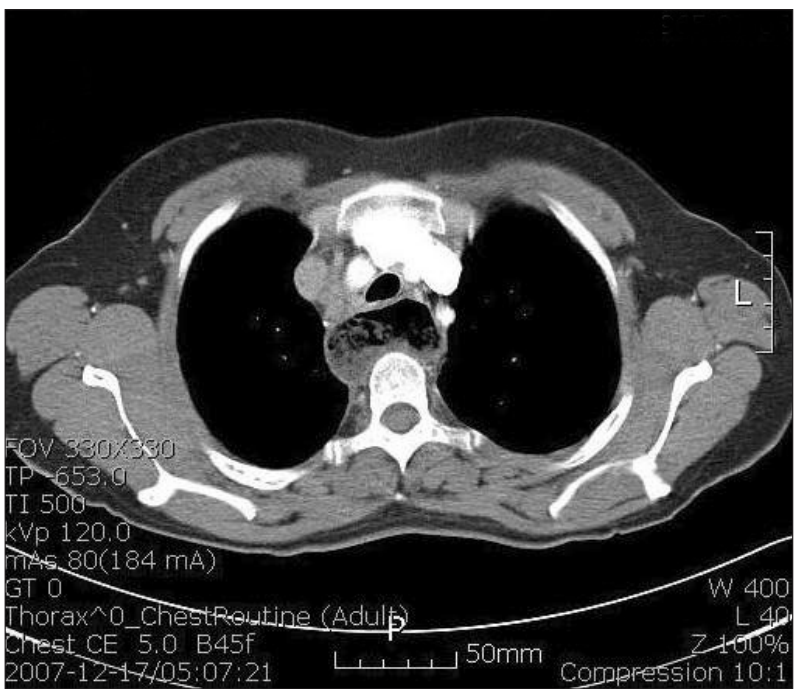

Fig. 1. Preoperative contrast-enhanced chest computed tomographic (CT) scan shows food materials in the markedly dilated esophagus.

esophagus, and is a liability for lung aspiration [2].

Sarcoidosis is a systemic inflammatory disease that is characterized by the presence of non-caseating granulomas. While sarcoidosis can occur in any organ of the body, mediastinal lymph nodes and lungs are the most commonly involved organs [2]. Though extremely rare, esophageal involvement of sarcoidosis can also occur. In such cases, achalasia-like dysmotility may occur [3]. Even though the preoperative CT showed esophageal dilatation, we the authors omissively overlooked the possibility of pulmonary aspiration and that sarcoidosis in rare occasions has an association with achalasialike dysmotility, and were blinded by the fact that the patient did not show or complain of any distinctive signs or symptoms of achalasia and the fact that the patient had no oral intake for at least 11 hours prior to induction. According to Sezgin et al. [4], for 35 patients who were fasting with achalasia, 28 presented with esophageal dilatation, lower esophageal stenosis and food retention on the ultra-sonogram. And these findings coincide with our current case, in which esophageal dilatation $(5.2 \times 4.3 \mathrm{~cm}$ in size $)$ and food retention were identified on the preoperative CT (Fig. 1).

In conclusion, a clear understanding of the risk factors is crucial in preventing further cases of pulmonary aspiration. This experience tellingly reminds us that even with no or little symptoms of achalasia, the risk of pulmonary aspiration should not be ignored when inducing anesthesia in patients with or suspicion of achalasia. An appropriate fasting time and premedication with $\mathrm{H} 2$ receptor antagonists or pro-kinetics is recommended to reduce the amount of and lower the acidity of the gastro-esophageal contents. Prior to induction of anesthesia, insertion of a naso-gastric tube can be used to ascertain the presence of food in the esophagus and stomach, and a $\mathrm{pH}$ measurement of the gastric contents with the immediate removal of aspirants could also be helpful for the prediction of prognosis and improve the outcome. Once pulmonary aspiration has occurred, active removal of the aspirated material guided by a FOB will also lower the morbidity of aspiration pneumonia.

\section{References}

1. Warner MA, Warner ME, Weber JG. Clinical significance of pulmonary aspiration during the perioperative period. Anesthesiology 1993; 78: 56-62.

2. Goyal RK. Disease of the esophagus. In: Harrison's principles of internal medicine. 16 th ed. Edited by Kasper DL, Braunwald E, Fauci AS, Hauser SL, Longo DL, Jameson JL, et al. New York, McGraw-Hill publishing. 2008, pp 1847-50, 2135-9.

3. Bredenoord AJ, Jafari J, Kadri S, Simcock DE, Sifrim D, Preston SL. Case report: achalasia-like dysmotility secondary to oesophageal involvement of sarcoidosis. Gut 2011; 60: 153-5.

4. Sezgin O, Ulker A, Temuçin G. Sonographic findings in achalasia. J Clin Ultrasound 2001; 29: 31-40. 